\title{
HACIA EL DESARROLLO DE LAS COMPETENCIAS DIGITALES DE LA UNESCO DURANTE EL CONFINAMIENTO EN UN CURSO DE INTERACCIÓN PERSONA- COMPUTADOR
}

\section{TOWARDS THE DEVELOPMENT OF UNESCO'S DIGITAL SKILLS DURING CONFINEMENT IN A HUMAN- COMPUTER INTERACTION COURSE}

\author{
Gabriel Elías Chanchí Golondrino, \\ Manuel Alejandro Ospina Alarcón², \\ Mónica Esther Ospino Pinedo 3
}

1 Gabriel Elías Chanchí Golondrino. Doctor en Ingeniería Telemática - Universidad del Cauca. Docente Facultad de Ingeniería - Universidad de Cartagena. Colombiano, C.C. 10.301.274. gcchanchig@unicartagena.edu.co. ORCID: https://orcid.org/0000-0002-0257-1988.

2 Manuel Alejandro Ospina Alarcón. Doctor en Ingeniería - Ciencia y Tecnología de Materiales - Universidad Nacional de Colombia -sede Medellín. Docente - Facultad de Ingeniería - Universidad de Cartagena. Colombiano, C.C. 71.265.598. mospinaa@unicartagena.edu.co. ORCID: https://orcid.org/0000-0003-4510-0753.

3 Mónica Esther Ospino Pinedo. Magister en Dirección Estratégica en Tecnologías de Información y Orientación Empresas de Software - Universidad Internacional Iberoamericana. Docente - Facultad de Ingeniería - Universidad de Cartagena. Colombiana, C.C. 45.758.598. mospinop@unicartagena.edu.co. ORCID: https://orcid.org/0000$\underline{0003-3357-6316 .}$.

\section{RESUMEN}

Como consecuencia del confinamiento provocado por el COVID-19, las Instituciones de Educación Superior han adaptado sus procesos académicos a las particularidades de la denominada presencialidad remota, teniendo como desafío en los diferentes cursos, el desarrollo de las competencias prácticas en los estudiantes y de las competencias digitales en los docentes. En este orden de ideas, la UNESCO partiendo de la visión de la agenda para el desarrollo sostenible, ha propuesto un marco de competencias TIC para docentes, el 
cual comprende un conjunto de 18 competencias estructuradas en 3 niveles y 6 aspectos. Este artículo propone como contribución el desarrollo de competencias digitales de la UNESCO, dentro de una de las temáticas prácticas del curso electivo de Interacción PersonaComputador del Programa de Ingeniería de Sistemas de la Universidad de Cartagena. De manera específica, se realizó la adaptación del tema "test con usuarios" del curso, el cual tradicionalmente es desarrollado de manera práctica en un laboratorio de usabilidad. De este modo, usando herramientas de teleconferencia, de seguimiento remoto y de gestión de encuestas, los estudiantes y el profesor del curso conformaron la infraestructura básica de un laboratorio virtual de usabilidad, en el cual los estudiantes desarrollaron las prácticas de la temática en mención.

PALABRAS CLAVE: Competencias digitales, COVID-19, IPC, test con usuarios, usabilidad.

\section{ABSTRACT}

As a consequence of the confinement caused by COVID-19, Higher Education Institutions have adapted their academic processes to the particularities of the so-called remote presence, having as a challenge in the different courses, the development of practical skills in students and digital skills in teachers. In this order of ideas, UNESCO, starting from the vision of the agenda for sustainable development, has proposed a framework of ICT competencies for teachers, which comprises a set of 18 competencies structured in 3 levels and 6 aspects. This article proposes as a contribution, the development of digital competences of UNESCO, within one of the practical topics of the elective course of Human-Computer Interaction of the Systems Engineering Program of the University of Cartagena. Specifically, the topic "user testing" was adapted from the course, which is traditionally developed in a practical way in a usability laboratory. In this manner, using teleconferencing, remote monitoring and survey management tools, the students and the course teacher set up the basic infrastructure of a virtual usability laboratory, in which the students could develop the practices of the topic in question.

KEYWORDS: Digital skills, COVID-19, $\mathrm{HCl}$, user testing, usability.

\section{INTRODUCCIÓN}

La pandemia originada por el COVID-19 ha generado cambios significativos en diferentes sectores como la economía, la educación, la industria, la salud, entre otras, las cuales han tenido que adaptar sus diferentes procesos a las dinámicas exigidas por la virtualidad (Alemán et al., 2020; Cho \& Hong, 2021; Constantino \& Neumeyer, 2020). En este sentido, a nivel educativo son diferentes los desafíos que han tenido que ser abordados, tales como: la brecha digital, el manejo de competencias digitales por parte de los docentes, la adaptación de las metodologías para desarrollo de las clases y evaluaciones y la adaptación de otros procesos inherentes al contexto educativo, como es el caso de los eventos académicos, las reuniones y los aspectos administrativos (Álvarez et al., 2020; Chanchí et al., 2020; Chanchí \& Hernández, 2020; García et al., 2020; Murillo \& Duk, 2020).

Uno de los desafíos de la presencialidad remota ocasionada por el COVID-19, ha sido el desarrollo de las competencias prácticas y digitales por parte de profesores y estudiantes, en diferentes cursos teórico-prácticos o meramente prácticos (Aguirre \& Yupa, 2020; Martínez \& Garcés, 2020). De acuerdo con la agenda 2030 para el desarrollo sostenible aprobada por la Asamblea General de las Naciones Unidas, que se orienta a la construcción de sociedades del conocimiento inclusivas, la UNESCO propuso un marco de competencias TIC para docentes, donde incluye un conjunto de 18 competencias organizadas en 3 niveles 
y 6 aspectos. Este marco ha sido ideado con el objetivo de preservar las competencias que siguen siendo pertinentes y enmarcarlas en el contexto de los actuales avances tecnológicos y las exigencias cambiantes de la vida y el trabajo (UNESCO, 2019). En la Figura 1 se muestran las 18 competencias con sus 6 aspectos y sus 3 niveles.

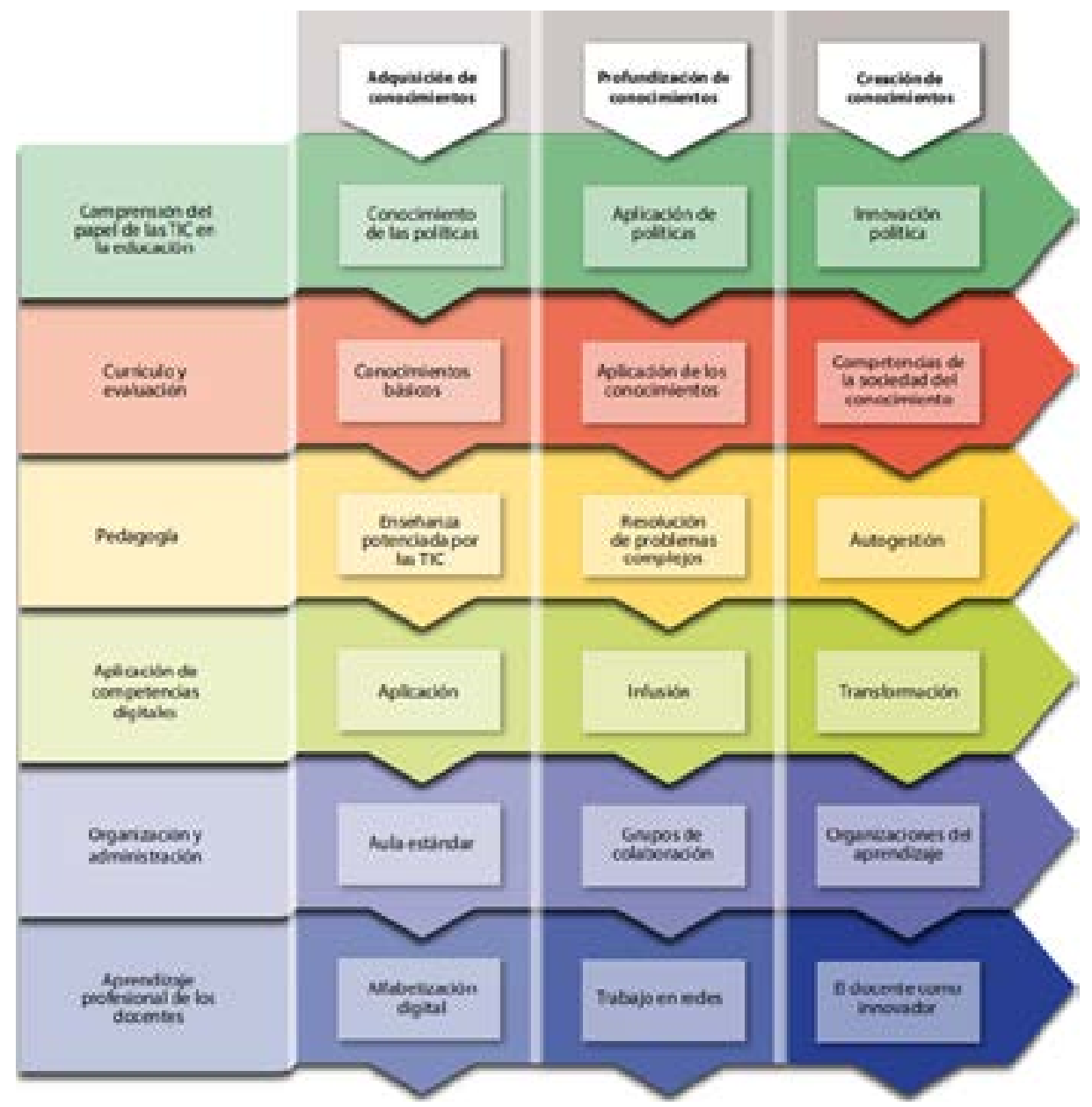

Figura 1 - Marco de competencias de la UNESCO (UNESCO, 2019)

En este sentido, uno de los cursos que incluye el desarrollo de competencias prácticas es el de Interacción Persona Computador (IPC) del Programa de Ingeniería de Sistemas de la Universidad de Cartagena. La disciplina de IPC es un área multidisciplinaria y emergente que se ubica en la intersección entre la psicología cognitiva, la ingeniería de las aplicaciones ergonómicas, las ciencias sociales y la informática aplicada, cuyo objetivo es proveer una comprensión sobre la forma en la que los usuarios trabajan, y la manera en la que los sistemas computacionales y sus interfaces requieren ser diseñadas para facilitar el logro de sus tareas (D'adamo et al., 2011). El curso de IPC desarrollado en el programa y en la institución, comprende en términos generales las temáticas relacionadas con el diseño y 
evaluación de sistemas interactivos desde la perspectiva de la usabilidad y la accesibilidad

Una de las temáticas más relevantes del curso de IPC es la de pruebas o test con usuarios, que incluye la estructuración, diseño y ejecución de pruebas de usabilidad en un entorno controlado (conocido como laboratorio de usabilidad) (Chanchí et al., 2018; Delgado et al., 2019). Dentro de la presencialidad, los estudiantes se encargaban de conformar mediante herramientas hardware y software un entorno de pruebas en los laboratorios de informática de la Universidad, dentro del cual cada grupo debía evaluar la usabilidad de un aplicativo software (aplicaciones de escritorio, páginas web comerciales, aplicaciones móviles, etc) con al menos 5 usuarios externos al curso, de acuerdo a las recomendaciones de Nielsen (Barnum et al., 2003; Nielsen, 2000). A partir de lo anterior, las dinámicas exigidas por la presencialidad remota obligaron al desarrollo de competencias digitales por parte de los docentes del curso y los estudiantes, de cara a la adaptación de las diferentes temáticas y en especial de la temática práctica de las pruebas de usabilidad, con el fin de propiciar el cumplimiento de las competencias prácticas del curso, de manera semejante a la forma en la que éstas se desarrollaban durante la presencialidad.

Así, este artículo propone como contribución el desarrollo de competencias digitales dentro del curso de IPC, de cara a la adaptación de las actividades prácticas. De manera específica, con el apoyo de los estudiantes se diseñó e implementó una infraestructura de laboratorio virtual de usabilidad para la realización de pruebas de usuario, sobre diferentes tipos de software. En este sentido, el desarrollo de competencias digitales en el curso de IPC se circunscribe dentro del marco de competencias digitales propuesto por la UNESCO (ver Figura 1), dado que al aporte realizado en el curso conllevó la adquisición (conceptualización de las pruebas de usuario), profundización (diseño y adaptación de un entorno virtual de pruebas) y creación de conocimiento (desarrollo de pruebas de usuario con apoyo del entorno) alrededor de la temática de pruebas de usabilidad. Este artículo pretende servir de referencia en cuanto al desarrollo de las competencias prácticas por parte de los cursos de las Facultades de Ingeniería del país. Por otra parte, el diseño de la infraestructura virtual propuesta servirá de apoyo para replicar y conducir pruebas de usuario tanto en entornos académicos como empresariales.

El resto del artículo está estructurado de la siguiente forma: en la sección 2 se presentan diferentes trabajos relacionados que sirven como referencia para este artículo; en la sección 3 se describe la metodología que se consideró para esta investigación; en la sección 4 se presentan algunos conceptos que se consideraron para este trabajo; seguidamente en la sección 5 se presenta el desarrollo de las competencias digitales dentro del curso IPC; finalmente en la sección 6 se describe las conclusiones y trabajos futuros derivados de la presente investigación.

\section{TRABAJOS RELACIONADOS}

Son varios los estudios llevados a cabo para implementar estrategias digitales en época de la pandemia. En (Expósito \& Marsollier, 2020) los autores evaluaron un conjunto de estrategias, recursos pedagógicos y tecnológicos usados por docentes argentinos dentro del modelo de educación virtual, implementado durante el confinamiento preventivo y cierre de establecimientos educativos. El estudio fue realizado mediante la aplicación de un instrumento cuantitativo sobre un total de 777 personas, en su mayoría profesores de diferentes instituciones de Mendoza-Argentina. El estudio desarrollado permitió evidenciar las desigualdades existentes en cuanto al uso de tecnologías y recursos pedagógicos digitales. 
Estas diferencias, son manifestadas en relación con el tipo de gestión, el nivel educativo, la situación socioeconómica de los alumnos, el rendimiento académico y el apoyo por parte de la familia del estudiante.

(Chang et al., 2021) presentan una recopilación de experiencias académicas desarrolladas en diferentes facultades de odontología pertenecientes a varios países asiáticos. Esta recopilación fue realizada a partir de las contribuciones presentadas en tres simposios virtuales organizados por la Asociación Odontológica de Ciencias de China-Taipei. Como resultado de los aportes realizados en los eventos se obtuvo que en su mayoría las clases se desarrollaron de manera remota haciendo uso de herramientas tales como ZOOM, Google Meet y Skype. Del mismo modo en la mayoría de las facultades las clases desarrolladas mediante el uso de laboratorios tuvieron un porcentaje alto de deserción. Como conclusiones del estudio, los autores consideraron que dada la importancia de las prácticas de laboratorio en los cursos de odontología, es importante promover el desarrollo de sistemas basados en realidad virtual, que permitan apropiar de mejor forma las temáticas en los estudiantes.

(Almetwazi et al., 2020) analizan las experiencias y enseñanzas adquiridas dentro del programa de farmacia de la Universidad del Reino Saudi durante la pandemia. Los autores abordan diferentes aspectos relacionados con la transición a nivel de las clases, las prácticas de laboratorio, los modelos de evaluación y las actividades extracurriculares. A nivel de las clases, dentro del programa en mención se desarrollaron sesiones virtuales con apoyo de un sistema de gestión de aprendizaje (Blackboard), contando con el soporte de la universidad a través de un canal de youtube y asesorías en línea. Del mismo modo, dada la importancia de las prácticas de laboratorio dentro del programa, en primera instancia se generaron contenidos de video por parte de los profesores con la conceptualización y el desarrollo de estas, los cuales fueron dirigidos a los estudiantes de la facultad. Por otra parte, como complemento a los contenidos generados, se desarrollaron prácticas presenciales en grupos pequeños de estudiantes.

(Jabbar et al., 2021) examinan las experiencias y enseñanzas obtenidas en los diferentes programas de la Escuela de Veterinaria de Melburne. En este sentido se presenta la forma en la que abordaron tanto las clases teóricas, como las clases prácticas y teórico-prácticas de los programas. A nivel de la parte teórica de los cursos, los profesores proporcionaron diferentes lecturas que dispusieron dentro del sistema de gestión de aprendizaje (Canvas) e implementaron en la metodología de aula invertida, la cual desarrollaron en los encuentros virtuales. Por otra parte a nivel de los componentes prácticos de los cursos, se hizo uso de recursos educativos disponibles en formato de video, dando prioridad a aquellos generados en forma de tutorial mediante herramienta Loom. Como desafíos a futuro, los autores plantean la necesidad de explorar otras alternativas para hacer la experiencia más interactiva, como es el caso de la realidad virtual.

(Cotino, 2021) muestra un análisis sobre la forma en la que diferentes países han abordado la emergencia sanitaria, desde el punto de vista de la educación. En este sentido, los autores resaltan que aunque la educación digital era considerada como excepcional o complementaria a la educación presencial, la decisión de los países de dar continuidad a los procesos educativos de manera remota durante el confinamiento ha hecho que ahora esta tome un rol protagónico y genere nuevos desafíos para las instituciones educativas. Así, dentro de los desafíos que se han encontrado se evidencia la superación de la brecha digital, el fomento de 
las competencias digitales y el pensamiento computacional en profesores y estudiantes.

(Cifuentes, 2020) realizó un análisis sobre la forma en la que la pandemia ha generado cambios necesarios en los procesos académicos, y la manera en la que profesores y estudiantes han tenido que adaptarse rápidamente a la educación a distancia y al uso de las nuevas tecnologías para el desarrollo de las clases. Así mismo, se resalta que aunque desde hace mucho tiempo se ha venido promulgando la importancia de la tecnología en el contexto educativo, los cambios exigidos por la presencialidad remota han sorprendido a los diferentes miembros de la comunidad académica. Como posible solución a los desafíos mencionados, el autor propone formar al profesorado en el aprovechamiento de las TIC, así como adaptar las metodologías para el desarrollo de las sesiones virtuales de los cursos. Finalmente, se presenta un caso de estudio sobre el desarrollo de las actividades académicas de un curso de Economía de la Universidad de Murcia.

(Jordan et al., 2021) revisan diferentes limitaciones y oportunidades en cuanto al uso y aplicación de la educación tecnológica como apoyo a los procesos académicos durante el periodo de presencialidad remota. En este sentido, dentro de los desafíos identificados se encuentra que: no todos los currículos pueden adaptarse a las dinámicas del aprendizaje en línea; la ansiedad de los estudiantes por conocer su progreso escolar; las brechas a nivel de infraestructura y a nivel de capacitación de los docentes por aprovechar las tecnologías en el desarrollo de las actividades académicas. De la misma manera, dentro de las posibles oportunidades se encuentran: la creación de plataformas para albergar recursos educativos a nivel global que puedan ser aprovechados por profesores en diferentes partes del mundo; hacer uso de medios como la televisión y la radio mediante tecnologías de fácil acceso para llegar a estudiantes con falencias en la conectividad; el uso de tecnologías como los SMS y WhatsApp para brindar soporte a los estudiantes; finalmente el aprovechamiento de recursos educativos abiertos como complemento a las actividades extracurriculares.

En (Murillo \& Duk, 2020) los autores hacen una reflexión sobre los cambios originados por la pandemia en los procesos académicos de las diferentes instituciones educativas de Latinoamérica. Expresan que la transición hacia la presencialidad remota ha evidenciado dos problemáticas principales: la brecha a nivel de infraestructura tecnológica y la brecha digital. Con respecto a la primera, mencionan que existen muchos estudiantes que se han quedado al margen de la virtualidad debido a las carencias en cuando a infraestructura de acceso, algo que en Latinoamérica se debe prestar atención considerando que solo 4 de cada 10 hogares cuenta con acceso a internet. Por otra parte, con respecto a la brecha digital se menciona que son pocos los profesores que manejan las tecnologías con sentido pedagógico, al igual que son minoría los que saben cómo diseñar procesos de aprendizaje en entornos virtuales. Del mismo modo, concluyen que las instituciones educativas de Latinoamérica en su mayoría no cuentan con las competencias necesarias para asumir el desafío de la virtualidad

(Kem, 2020) aborda las estrategias implementadas en el Departamento de Lenguas Extranjeras de la Escuela Normal Superior adscrita a la Universidad de Yaunde I de Camerún, como respuesta a los cambios académicos suscitados por el confinamiento. En primera instancia, los autores resaltan que la transición hacia la educación virtual ha tenido como desafío la superación de la brecha a nivel de la infraestructura y a la formación de los docentes y personal académico en cuanto a las competencias digitales. Respecto a las estrategias implementadas en la unidad 
académica en mención, se describe la implementación de una plataforma virtual para el seguimiento de las actividades académicas como repositorio del material de clase de los diferentes cursos. Así mismo, el uso de redes sociales como alternativa para soporte y acompañamiento a los estudiantes en sus diferentes cursos. Finalmente, se menciona la importancia de fomentar las competencias digitales de cara a enriquecer las metodologías para el desarrollo de los cursos de manera virtual.

En (Velásquez \& Ruidiaz, 2020) los autores realizan una reflexión sobre la adaptación de los procesos académicos desde la presencialidad remota. A partir de lo anterior, los autores resaltan como principales limitaciones la brecha tecnológica evidenciada en la dificultad de muchos estudiantes para acceder a las sesiones virtuales, así como las falencias en cuanto a las competencias digitales por parte de los profesores para aprovechar las tecnologías de la información y adaptar las metodologías a las dinámicas exigidas por la virtualidad. Finalmente, los autores resaltan como oportunidades para las instituciones educativas: la posibilidad de impulsar otros tipos de aprendizaje, otro tipo de herramientas para apropiar las temáticas de los cursos e innovar en las metodologías tradicionales de enseñanza-aprendizaje.

Los trabajos presentados en esta sección dan cuenta de un conjunto de retos importantes que se han obtenido desde las experiencias en diferentes partes del mundo, a partir del desarrollo de actividades académicas durante el confinamiento. Uno de los retos más relevantes y que se aborda en el presente artículo es la formación de los docentes en cuanto al desarrollo de competencias digitales, de tal modo que se aproveche al máximo el potencial de las tecnologías de la información en la apropiación de las diferentes temáticas comprendidas en los cursos de cada programa académico.

\section{METODOLOGÍA}

Con el objetivo de desarrollar las competencias prácticas dentro de la temática de pruebas de usuario del curso de IPC del Programa de Ingeniería de Sistemas de la Universidad de Cartagena, se definieron 4 fases metodológicas a saber: caracterización de las pruebas de usuario, exploración y selección de tecnologías, conformación de escenario controlado, desarrollo de pruebas de usabilidad (ver Figura 2).

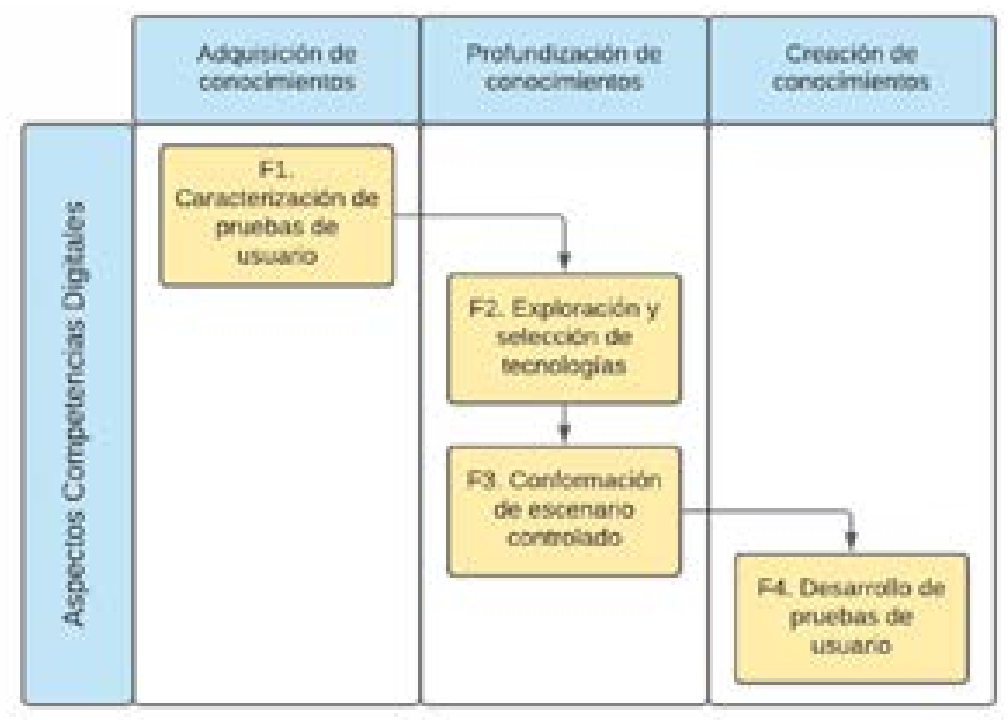

Figura 2 - Metodología considerada 
Las cuatro fases de la Figura 2, se encuentran dentro del marco de competencias propuesto por la UNESCO mostrado en la Figura 1. La fase 1 de la metodología está articulada al nivel de adquisición de conocimientos, las fases 2 y 3 están comprendidas dentro del nivel de profundización de conocimiento, mientras que la fase 4 corresponde al nivel de creación de conocimientos. El desarrollo de estas fases involucra de manera transversal la comprensión del papel de las TIC, la apropiación del currículo, la adaptación del modelo pedagógico y aspectos relacionados con el estudio del currículo, la adaptación del modelo pedagógico, la formación del docente, entre otros aspectos que están comprendidos en el marco de competencias digitales de la UNESCO.

En la fase 1 se conceptualizan y caracterizan las diferentes etapas que componen una prueba de usuario, así como los posibles requerimientos tecnológicos para el desarrollo de estas. En la fase 2 a partir de los requerimientos tecnológicos identificados se explora un conjunto de tecnologías por parte del docente del curso y los estudiantes para la conformación del entorno controlado. En la fase 3 a partir de las herramientas identificadas en la fase 2 , se diseña y conforma un laboratorio virtual para la ejecución de pruebas de usabilidad. Finalmente, en la fase 4, los estudiantes del curso desarrollaron de manera grupal diferentes prácticas de usabilidad sobre portales web de carácter comercial.

\section{RESULTADOS}

En esta sección se presentan los resultados de la aplicación de las competencias digitales dentro del curso de IPC. De acuerdo con la metodología considerada, en primera instancia se presenta la caracterización de las pruebas de usabilidad, la cual incluye la conceptualización y estructura de las pruebas de usabilidad (fase 1). posteriormente, se presenta el diseño y conformación del laboratorio de usabilidad, a partir de herramientas tecnológicas (fases $2 \mathrm{y}$ 3). Finalmente se muestra un caso de aplicación sobre el uso del entorno de pruebas desarrollado (fase 4).

\subsection{Caracterización de pruebas de usabilidad}

Con el incremento en el número de aplicaciones desarrolladas en el mercado, una de los aspectos claves para garantizar la calidad del software de cara a garantizar el diseño centrado en el usuario es la usabilidad (Arroqui et al., 2016; Ling \& Chen, 2011; Macaulay et al., 2009; Yaohua \& Zhengjie, 2006). De acuerdo a la ISO 9241-11, la usabilidad puede definirse como el grado en el que un producto software puede ser usado por usuarios específicos para conseguir metas específicas con efectividad, eficiencia y satisfacción en un contexto de uso específico (Ferreira et al., 2020; Finstad, 2010; Martins et al., 2013; Retnani et al., 2018; Weichbroth, 2020). Del mismo modo, de acuerdo a la ISO 9126, la usabilidad de un producto software puede ser definida en términos de atributos tales como la facilidad de aprendizaje, la capacidad de recordación, facilidad de operación y capacidad de resultar atractivo (Dzulfiqar et al., 2019; González et al., 2012; Santos et al., 2016; Zeman et al., 2009). La facilidad de aprendizaje y la capacidad de operación están relacionados con la eficiencia, la facilidad de operación está relacionada con eficacia, mientras la capacidad de resultar atractivo está directamente relacionada con la satisfacción.

Para evaluar la usabilidad de un producto software se suele hacer uso de los atributos de eficacia, eficiencia y satisfacción de la norma ISO 9241-11, en el marco de un test con usuarios desarrollado en un entorno controlado o laboratorio de usabilidad (Delgado et al., 2018). Un laboratorio de usabilidad puede ser definido como un lugar compuesto generalmente de dos espacios físicos, uno 
para el usuario que evalúa e interactúa con el software y otro para los coordinadores de la prueba, los cuales observan el comportamiento y el cumplimiento de las tareas por parte del usuario. En este espacio físico, se hacen evaluaciones de usabilidad y de accesibilidad a productos, sistemas y dispositivos, por medio de software y equipos hardware especializados en el monitoreo y registro de las acciones del usuario con el producto a evaluar. Este set de herramientas hardware y software de las que dispone el laboratorio de usabilidad, debe facilitar la obtención de indicadores de los atributos de eficiencia, eficacia y satisfacción (Albornoz et al., 2019).

En una prueba de usuario convencional realizada en un laboratorio de usabilidad, un grupo de coordinadores se encarga de observar de manera silenciosa, el desarrollo de un conjunto de tareas por parte de un usuario, mientras éste interactúa con un software determinado (ver Figura 3). A partir de la observación realizada en la prueba, los coordinadores buscan obtener la eficacia, la eficiencia y la satisfacción en el uso del producto software evaluado. La eficacia puede ser determinada a partir del número de tareas cumplidas de manera exitosa, la eficiencia puede calcularse en términos del tiempo empleado en el desarrollo de las tareas, mientras que la satisfacción puede obtenerse a partir del uso de encuestas de satisfacción y la observación de los gestos del usuario mientras interactúa con un aplicativo software (Chanchi et al., 2020; Delgado et al., 2018; Enriquez \& Casas, 2013).

Un test con usuarios está conformado por varias etapas (previa selección de los usuarios y definición de los instrumentos de la prueba) dentro de las cuales se encuentran (ver Figura 4): acuerdo de confidencialidad, cuestionario pretest, listado de tareas y finalmente cuestionario pos-test. En el acuerdo de confidencialidad se le solicita autorización al usuario para la captura de los datos en la prueba y se le informa que sus datos se usarán solamente para propósitos académicos; en el cuestionario pre-test se le solicita al usuario diligenciar los datos relacionados con su perfil; en el listado de tareas los usuarios deben realizar las tareas definidas sin contar con la intervención del coordinador; en el cuestionario pos-test, los usuarios diligencian un conjunto de preguntas de carácter cuantitativo y cualitativo una vez se ha terminado la ejecución de las tareas (Chanchí et al., 2018;

Delgado et al., 2019.

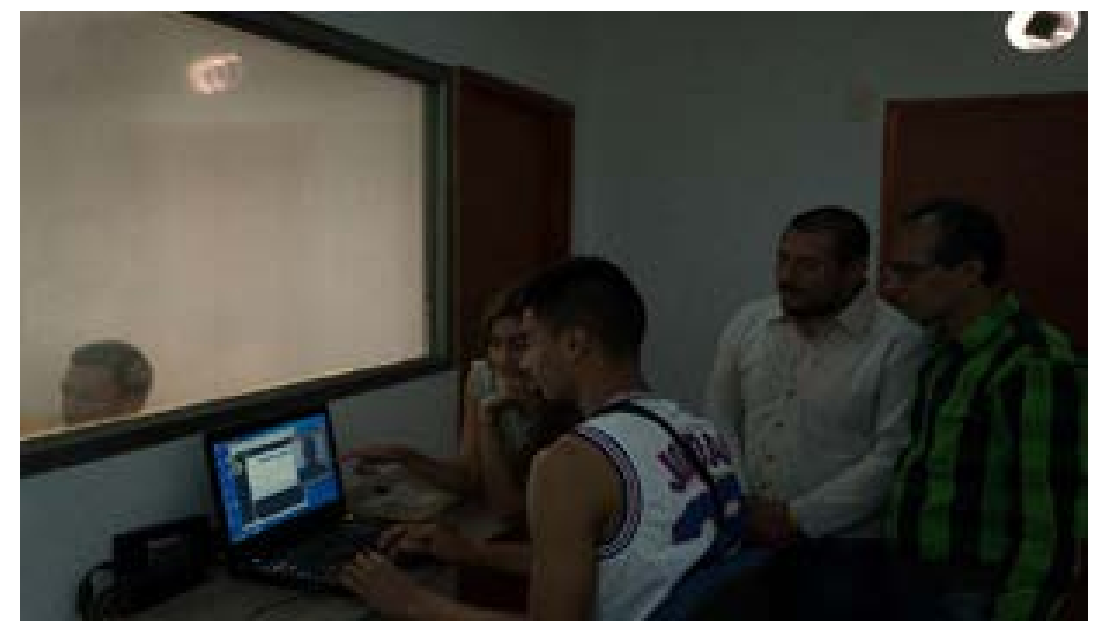

Figura 3 - Laboratorio de usabilidad 

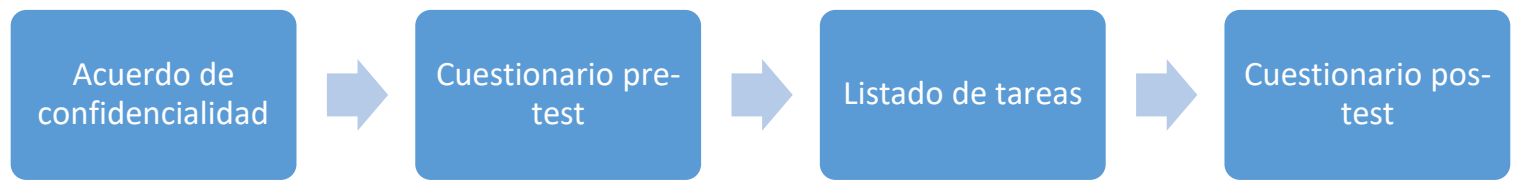

Figura 4 - Etapas de una prueba de usabilidad

A partir de lo presentado anteriormente, la adaptación en el desarrollo de las pruebas de usuario implica no solo proporcionar una infraestructura virtual para el seguimiento del usuario en una prueba y la determinación de la eficacia, eficiencia y satisfacción, sino también contar con un conjunto de herramientas que permitan diligenciar los diferentes instrumentos de la prueba.

\subsection{Diseño y conformación de escenario controlado}

Una vez fueron caracterizadas las pruebas de usabilidad, con apoyo de los estudiantes, se exploraron las herramientas más adecuadas para dar soporte al desarrollo de las pruebas de usuario de manera virtual, así como aquellas que servirán de apoyo para el diligenciamiento de los instrumentos asociados a la prueba de usuario. En este sentido, en la Figura 5 se presenta la estructura general del laboratorio de usabilidad virtual diseñado y propuesto dentro del curso de IPC.

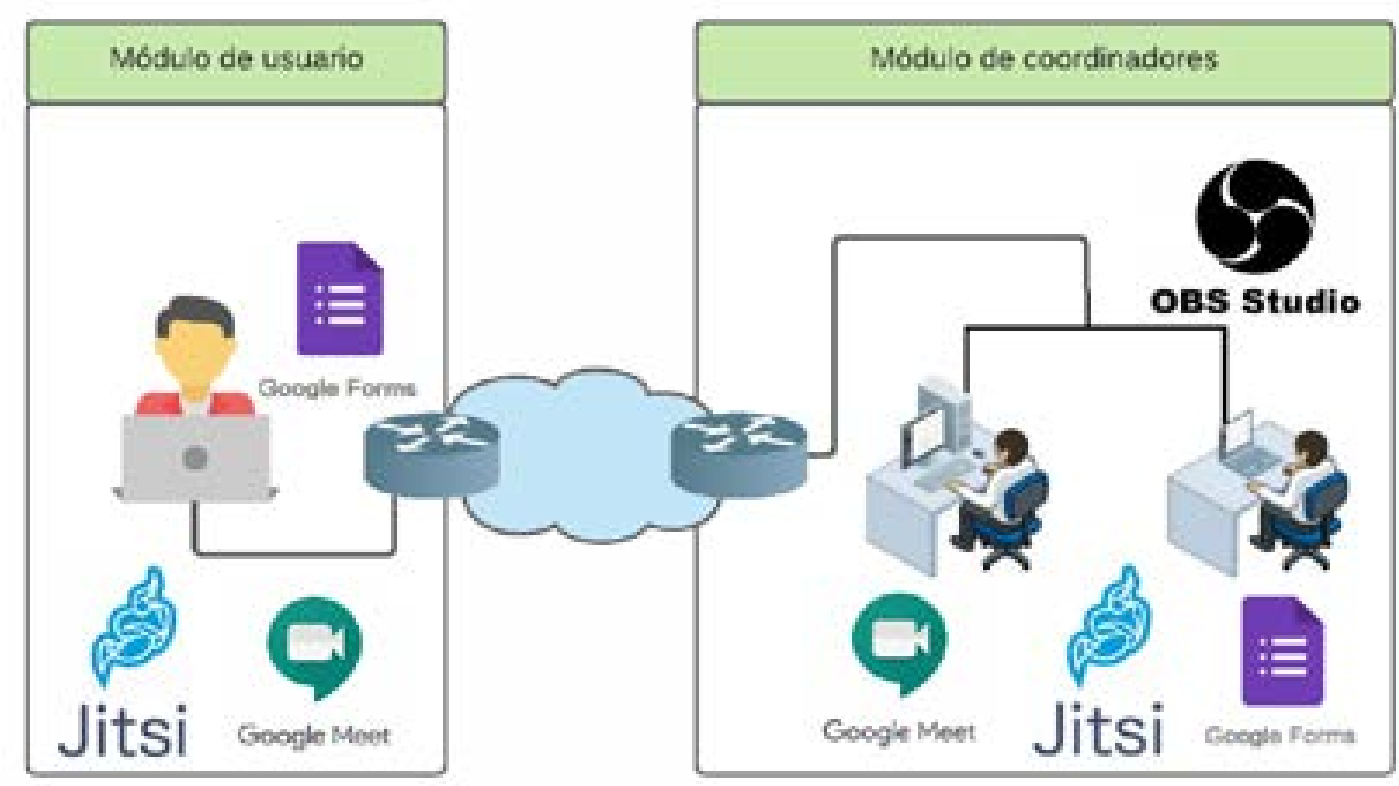

Figura 5 - Laboratorio de usabilidad virtual propuesto

En primer lugar, para la conformación de los instrumentos de evaluación de las pruebas de usuario se seleccionó la herramienta Google Forms, la cual permite la creación de formularios y cuestionarios basados en preguntas de selección múltiple. En este sentido, esta herramienta posibilitó la creación del acuerdo de confidencialidad, el cuestionario pre-test, 
el listado de tareas y el cuestionario pos-test, los cuales diligencia el usuario durante la prueba. En segundo lugar, en lo referente a la infraestructura del entorno controlado para el desarrollo de las pruebas, es importante mencionar que al igual que en la infraestructura física, la infraestructura virtual cuenta con dos módulos: el módulo de usuario y el módulo del coordinador o evaluador de la prueba, los cuales están conectados de manera remota. Se escogieron las herramientas Google Meet y Jitsi Meet para realizar el seguimiento virtual a las tareas que va realizando el usuario que hace la prueba sobre un determinado software o aplicación. En este sentido, es importante solicitar al usuario que comparta pantalla y encienda la cámara, lo anterior con el fin de verificar el cumplimiento de las tareas definidas en la prueba, así como realizar un análisis descriptivo sobre los gestos de los usuarios durante la interacción, de acuerdo con lo propuesto en (de Lera \& Garreta, 2008). Del mismo modo, con el fin de analizar y verificar a posteriori las diferentes interacciones realizadas por el usuario, se escogió la herramienta OBS Studio para grabar las diferentes sesiones de la prueba. Finalmente, es importante resaltar que la infraestructura propuesta en la Figura 5 posibilita la obtención de los atributos de eficacia, eficiencia y satisfacción, de cara a obtener el nivel de usabilidad del software evaluado por el usuario.

\subsection{Desarrollo de pruebas}

Con el fin de verificar la utilidad del entorno virtual diseñado y conformado, así como posibilitar el desarrollo de las competencias prácticas de los estudiantes del curso IPC, se conformaron un total de 9 grupos de trabajo, a cada uno de los cuales se les asignaron diferentes portales web de comercio electrónico a evaluar desde la perspectiva de la usabilidad. Dentro de los portales web evaluados por los estudiantes se encuentran: Ktronix, Surtitodo, Tienda del Músico, Tennis, Sigma Electrónica, Janus, Gef, Librería Panamericana y Vox Tienda Musical. A los estudiantes se les recomendó realizar la prueba con al menos 5 usuarios externos al curso sobre la infraestructura propuesta y diseñando los instrumentos de usabilidad mediante Google Forms, partiendo de los documentos base proporcionados por el profesor. Así, a modo de ejemplo, en la Figura 6 se presenta una captura de pantalla del escenario de pruebas, obtenida por el grupo que realizó la evaluación sobre el portal de Sigma Electrónica, el cual se dedica a la venta de productos electrónicos.

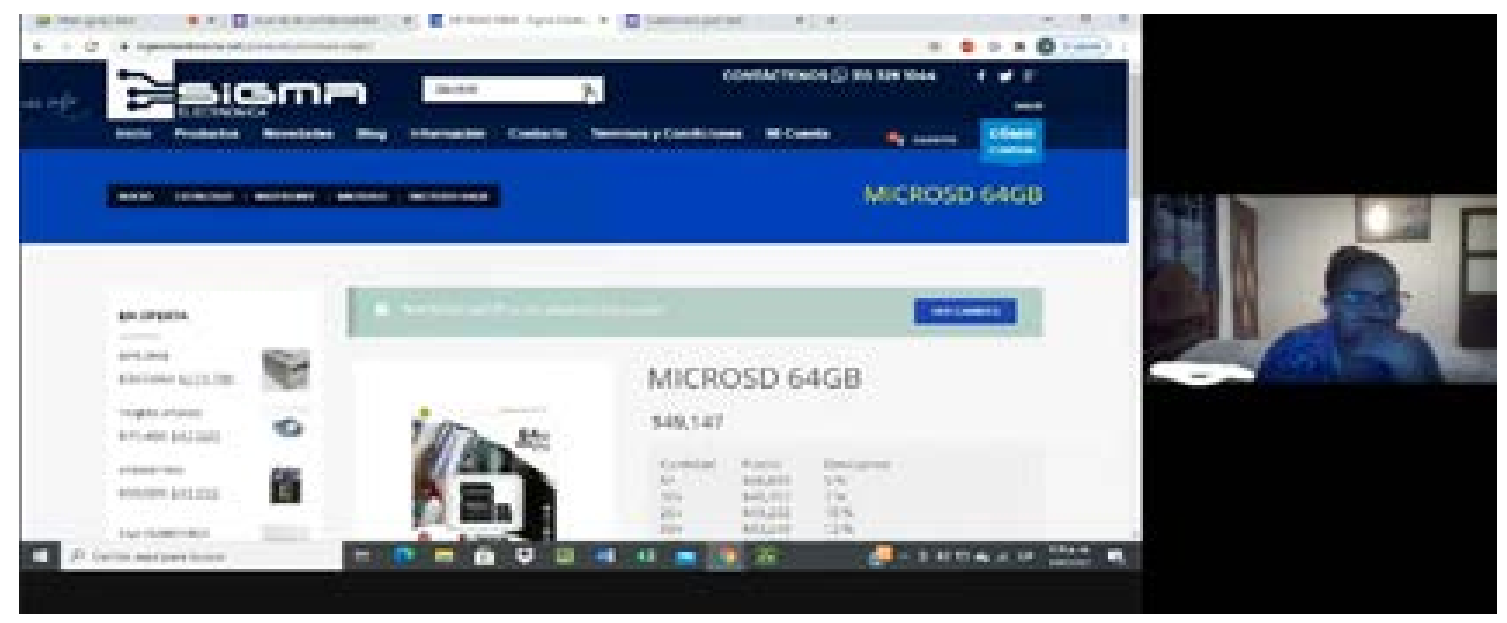

Figura 6 - Prueba de usabilidad sobre el portal de Sigma Electrónica 
El grupo de trabajo con el portal de Sigma Electrónica realizó la prueba con un total de 5 usuarios ( 3 mujeres y 2 hombres) con edades comprendidas entre los 22 y los 52 años, los cuales realizaron en total 3 tareas puntuales con sus respectivas sub-tareas dentro del portal web evaluado (diseñadas por el grupo de trabajo): realizar el registro en el portal, interactuar con el carrito de compras y finalmente realizar comentar en el blog del portal web. Con respecto a los atributos de eficacia, eficiencia y satisfacción obtenidos en la prueba, en la Tabla 1 se presenta el consolidado de los valores calculados por los estudiantes para dichos atributos para los 5 usuarios que participaron en la prueba (U1, U2, U3, U4, U5). El cálculo de la eficacia fue realizado a partir del porcentaje de sub-tareas realizadas de cada tarea por parte de los diferentes usuarios, de tal modo que un porcentaje del $100 \%$ implica el cumplimiento del total de las sub-tareas de cada tarea definida, mientras que un porcentaje menor a 100 hace referencia a que el usuario no pudo realizar alguna de las sub-tareas asociadas a las diferentes tareas (U1 y U5). En cuanto a la eficiencia, el cálculo es realizado a partir de la relación entre el tiempo estimado para cada tarea por parte de los evaluadores y el tiempo empleado por el usuario en realizar cada una de las tareas, de tal modo que si el tiempo empleado es menor al estimado, el porcentaje es mayor a 100 (U2 y U5) y si el tiempo empleado es mayor al estimado, el porcentaje es menor a 100. Finalmente, la satisfacción se obtiene a partir del promedio de las preguntas del cuestionario postest asociadas a la percepción del usuario con respecto al portal web, multiplicado por 20 para obtener el resultado en términos de porcentajes. Finalmente, al promediar los resultados de eficacia, eficiencia y satisfacción, se obtiene que el porcentaje o nivel de usabilidad para el portal Sigma Electrónica es de 91.74\%.

Tabla 1 - Resultados prueba de usabilidad grupo Sigma Electrónica

\begin{tabular}{|c|c|c|c|}
\hline Usuarios & Eficacia & Eficiencia & Satisfacción \\
\hline U1 & $88.88 \%$ & $75.85 \%$ & $46.6 \%$ \\
\hline U2 & $100 \%$ & $194.87 \%$ & $80 \%$ \\
\hline U3 & $100 \%$ & $95.71 \%$ & $56.6 \%$ \\
\hline U4 & $83.33 \%$ & $88.88 \%$ & $73.2 \%$ \\
\hline Promedio & $100 \%$ & $121.74 \%$ & $70 \%$ \\
\hline Nivel de usabilidad & $\begin{array}{c}\text { (Eficacia + } \\
\text { Eficiencia + } \\
\text { Satisfacción) / 3 } \\
\mathbf{9 1 . 7 5 4 \%}\end{array}$ & $115.54 \%$ & $65.26 \%$ \\
\hline
\end{tabular}

Finalmente, a partir de la observación realizada por los estudiantes sobre interacción de los usuarios con el portal web evaluado, se pudo concluir que aunque el portal ofrece una variedad de productos, tiene un tiempo de respuesta adecuado y es interactivo para el usuario final, es posible mejorar las opciones de búsqueda, en las cuales a la hora de pre- visualizar los resultados, muestra las imágenes de los diferentes productos que coinciden con el criterio de búsqueda, junto con el código asignado en la base de datos, lo cual puede hacer confundir a un usuario que no sea experto. Así mismo, dado que el propósito del portal es posibilitar la búsqueda y compra de productos, la barra de búsqueda puede hacerse más amplia. 


\subsection{Discusión de resultados}

Aunque las herramientas TIC se han venido difundiendo desde mucho tiempo antes de la pandemia originada por el COVID-19, la transición hacia la presencialidad remota ha tomado desprevenido a los diferentes miembros de la comunidad académica, en cuanto al aprovechamiento de las TIC en el contexto educativo. En este sentido, las competencias digitales propuestas por la UNESCO son una hoja de ruta para articular y adaptar de mejor forma los objetivos y competencias del currículo a las exigencias de la presencialidad remota. Lo presentado en este artículo pretende servir de referencia específica para desarrollar las competencias digitales de los docentes, como las competencias prácticas de los estudiantes dentro de del curso de IPC, así como cursos afines, como pueden ser los relacionados con la Ingeniería de Software.

De acuerdo con lo presentado en el estado del arte, para el desarrollo de las competencias prácticas en diferentes universidades del mundo, se optó por la grabación de videos prácticos por parte de los profesores, así como la realización de prácticas en grupos pequeños, dado que el uso de simuladores en algunos cursos meramente prácticos no tuvo la acogida por parte de los estudiantes. En el caso de la infraestructura virtual presentada en este artículo, demostró ofrecer incluso mejores ventajas a nivel de practicidad que las mostradas por las pruebas de usabilidad convencionales. Así, la efectividad de la infraestructura propuesta radica en la posibilidad de determinar la eficacia, la eficiencia y la satisfacción dentro de un test con usuarios, los cuales son factores claves en la determinación de la usabilidad según la norma ISO 9241-11.

Una de las oportunidades que develó la presencialidad remota es la de propiciar la generación de conocimiento a partir del trabajo en equipo entre estudiantes y docentes. En este sentido, la presencialidad remota dentro del curso de IPC permitió que tanto estudiantes como profesores trabajaran en conjunto para el diseño de un laboratorio virtual de usabilidad. Lo anterior se enmarca en los 3 niveles propuestos por las competencias: adquisición de conocimientos, profundización de conocimientos y creación de conocimientos. Estas enseñanzas llevan a repensar y adaptar las estrategias tradicionales empleadas para la evaluación de los estudiantes en el contexto universitario, pues, así como la presencialidad remota tiene desafíos, propicia también una mayor conectividad más allá de las sesiones virtuales.

Aunque en algunas universidades de Colombia el curso de IPC no se encuentra en los currículos de los programas de Ingeniería de Sistemas y son pocos las universidades que cuentan con la infraestructura de un laboratorio de usabilidad, lo propuesto en este artículo se constituye como un punto de partida a nivel metodológico y a nivel de infraestructura para la vinculación progresiva de temáticas como la usabilidad en los Programas de Ingeniería de Sistemas, con miras a la incorporación del curso de IPC en el currículo de estos programas. Del mismo modo la infraestructura propuesta, puede ser de gran interés por parte de las empresas para articular las pruebas de usabilidad en sus procesos de evaluación, con el fin de mejorar la calidad del software desarrollado en estas empresas.

\section{CONCLUSIONES Y TRABAJOS FUTUROS}

A partir de los desafíos de la presencialidad remota con respecto al desarrollo de las competencias para el aprovechamiento de las TIC por parte de docentes y estudiantes, este artículo propuso como aporte la apropiación de las competencias digitales propuestas por la UNESCO, dentro del curso IPC del Programa de Ingeniería de Sistemas de la Universidad de Cartagena. Se pretende servir de referencia 
en cuanto al desarrollo de competencias en diferentes cursos del contexto universitario, tomando como hoja de ruta el marco de competencias definidas por la UNESCO.

Uno de los desafíos a los que se enfrentaron los docentes en el desarrollo de cursos prácticos o teórico prácticos durante la presencialidad remota, fue adaptar estos cursos a las características de la virtualidad. En el caso puntual del curso de IPC, la temática de prueba de usuario exigía realizar pruebas con usuarios reales, razón por la cual dicha temática fue adaptada a partir del trabajo colaborativo entre estudiantes y docentes, con el fin de permitir que la virtualidad no afectara el cumplimiento de las competencias definidas en el microcurrículo del curso. En este sentido, este artículo propuso como aporte la conformación de una infraestructura de laboratorio de usabilidad para la conducción de pruebas convencionales de usabilidad.

La infraestructura del escenario virtual del laboratorio de usabilidad, reunió un conjunto de herramientas de fácil acceso que permiten tanto el diligenciamiento virtual de los instrumentos convencionales de una prueba de usuario, así como el seguimiento de las diferentes acciones que realiza el usuario mientras interactúa con un software determinado. Del mismo modo, estas herramientas permiten al coordinador de la prueba realizar un análisis sobre los gestos o actitudes que tiene el usuario mientras realiza las tareas definidas, lo cual puede dar cuenta de posibles aspectos en los que el software no genera la satisfacción adecuada al usuario.

Tanto la metodología de las pruebas, como la infraestructura presentada en este artículo pueden ser replicadas fácilmente por investigadores del área o empresas del mundo software teniendo presente la conducción de pruebas de usabilidad con el fin de identificar aquellos aspectos que pueden ser mejorados en el software y así retroalimentar su calidad. Cabe mencionar adicionalmente, que la infraestructura virtual optimiza los costos y resulta mucho más simple de desplegar que la infraestructura física, arrojando resultados iguales o mejores en cuanto a la obtención de niveles de usabilidad en un software determinado.

Las 9 pruebas realizadas por los diferentes grupos del curso de IPC sobre portales de comercio electrónico, permitieron concluir que la infraestructura conformada arroja resultados adecuados y permite desarrollar las competencias prácticas en los estudiantes, aportando así a cumplir con uno de los desafíos iniciales al abordar el curso de IPC en el modo de presencialidad remota. En este sentido, uno de los elementos claves es que la infraestructura permite obtener fácilmente los atributos de eficacia, eficiencia y satisfacción, los cuales son fundamentales para la obtención de los niveles de usabilidad de un software

Como trabajo futuro derivado del presente artículo, se pretende retroalimentar la infraestructura virtual base, de tal forma que no solo posibilite el desarrollo de pruebas sobre aplicaciones web y de escritorio, sino también sobre aplicaciones móviles.

\section{AGRADECIMIENTOS}

Los autores del artículo agradecen a la Universidad de Cartagena por el apoyo brindado en la presente investigación. Del mismo modo, los autores agradecen a los estudiantes del curso de IPC del programa de Ingeniería de Sistemas por la colaboración y los datos proporcionados para el presente artículo. 


\section{REFERENCIAS BIBLIOGRÁFICAS}

Aguirre, E., \& Yupa, B. (2020). Percepción de la educación virtual durante la Covid -19 en los colegios del Distrito Metropolitano de Quito, Ecuador, 2019-2020. Revista Del Grupo de Investigación En Comunidad y Salud A, 5, 63-76.

Albornoz, D. A., Moncayo, S. A., RuanoHoyos, S., Chanchí-Golondrino, G. E., \& Márceles-Villalba, K. (2019). Sistema software para la ejecución de pruebas de usabilidad bajo el enfoque de mouse tracking. TecnoLógicas, 22, 19-31. https:// doi.org/10.22430/22565337.1511

Alemán, I., Vera, E., \& Patiño-Torres, M. J. (2020). COVID-19 and medical education: Challenges and opportunities in Venezuela. Educacion Medica, 21(4), 272-276. https:// doi.org/10.1016/j.edumed.2020.06.005

Almetwazi, M., Alzoman, N., Al-Massarani, S., \& Alshamsan, A. (2020). COVID-19 impact on pharmacy education in Saudi Arabia: Challenges and opportunities. Saudi Pharmaceutical Journal, 28(11), 1431-1434. https://doi.org/10.1016/j. jsps.2020.09.008

Álvarez, H., Arias, H., Bergamaschi, A., López, Á., Noli, A., Ortiz, M., Pérez, M., RiebleAubourg, S., Rivera, M., Scannone, R., Vásquez, M., \& Viteri, A. (2020). La educación en tiempos de coronavirus. https://publications.iadb.org/publications/ spanish/document/La-educacion-entiempos-del-coronavirus-Los-sistemaseducativos-de-America-Latina-y-el-Caribeante-COVID-19.pdf
Arroqui, M., Mangudo, P., Pelliza, L., Murgolo, S., Ottonello, A., Ferragut, S., Rodriguez Alvarez, J., MacHado, C., \& Teyseyre, A. (2016). Combination of Agile Development and User Centered Design to Improve the Usability of a Beef-Cattle Farm Simulator. IEEE Latin America Transactions, 14(7), 3385-3392. https://doi.org/10.1109/ TLA.2016.7587646

Barnum, C., Bevan, N., Cockton, G., Nielsen, J., Spool, J., \& Wixon, D. (2003). The "Magic Number 5": Is It Enough for Web Testing? $\mathrm{CHI} 2003$ - Conference on Human Factors in Computing Systems, 698-699.

Chanchí, G. E., \& Hernández-Londoño, C. E. (2020). Estrategia basada en TIC para la organización de ferias virtuales de divulgación académica durante la pandemia de COVID-19. Revista ESPACIOS, 41(42), 66-80.

Chanchí, G. E., Ospina, M. A., \& Ospino, M. E. (2020). Análisis de sentimientos de la percepción de los estudiantes de Ingeniería de Sistemas de la Universidad de Cartagena (Colombia) sobre las actividades académicas desarrolladas durante el confinamiento debido al COVID-19. Revista Espacios, 41(42), 247259. https://doi.org/10.48082/espaciosa20v41n42p21

Chanchi, G. E., Ospina, M. A., \& Pérez, J. L. (2020). Sistema loT para la monitorización de la variabilidad del ritmo cardiaco en pruebas de usabilidad loT system to monitoring heart rate variability in usability tests. Revista Espacios, 41(25), 2020. https://www.revistaespacios.com 
Chanchí, G. E., Sánchez, M., \& Campo, W. Y. (2018). Sistema software para el análisis del estrés mental en test de usuarios. Campus Virtuales, 7(2), 105-114. www. revistacampusvirtuales.es

Chanchí G, G. E., Muñoz S, L. F., \& Campo M, W. Y. (2018). Proposal of a tool for the stimation of satisfaction in usability test under the approach of thinking aloud. Communications in Computer and Information Science, 944, 211-222. https:// doi.org/10.1007/978-3-030-03763-5_18

Chang, T. Y., Hong, G., Paganelli, C., Phantumvanit, P., Chang, W. J., Shieh, Y. S., \& Hsu, M. L. (2021). Innovation of dental education during COVID-19 pandemic. Journal of Dental Sciences, 16(1), 15-20. https://doi.org/10.1016/j.jds.2020.07.011

Cho, M. J., \& Hong, J. P. (2021). The emergence of virtual education during the COVID-19 pandemic: The past, present, and future of the plastic surgery education. Journal of Plastic, Reconstructive and Aesthetic Surgery. https://doi.org/10.1016/j. bjps.2020.12.099

Cifuentes, J. (2020). Docencia online y Covid-19 : la necesidad de reinventarse. Revista de Estilos de Aprendizaje, 13, 115-127. http:// revistaestilosdeaprendizaje.com/article/ view/2149/3248

Constantino, H., \& Neumeyer, A. (2020). Un marco conceptual para analizar el impacto económico de COVID-19 y sus implicaciones políticas. In PNUD America Latina y el Caribe (Issue 1). https://www. latinamerica.undp.org/content/rblac/es/ home/library/crisis_prevention_and_ recovery/a-conceptual-framework-foranalyzing-the-economic-impact-of-covi. $\mathrm{html}$
Cotino, L. (2021). La enseñanza digital en serio y el derecho a la educación en tiempos del coronavirus. Revista de Educación y Derecho, 21. https://doi.org/10.1344/ reyd2020.21.31283

D’adamo, M. H., Baum, A., Luna, D., \& Argibay, P. (2011). Interacción ser humanocomputadora: usabilidad y universalidad en la era de la información. Revista Del Hospital Italiano, 31(4), 123-130. https://www.hospitalitaliano.org.ar/ multimedia/archivos/noticias_attachs/47/ documentos/11426_123-130_HI4-13 DAdamo.pdf

de Lera, E., \& Garreta-Domingo, M. (2008). 10 heurísticos emocionales - pautas para evaluar la dimensión afectiva de los usuarios de forma fácil y económica. Revista Faz, 68-81. http://www.revistafaz. org/articulos_2/06_diezheuristicos_ delera_garreta.pdf

Delgado, D. M., Girón, D. F., Chanchí, G. E., \& Márceles, K. (2018). Propuesta de una herramienta para la estimación de la satisfacción en pruebas de usuario, a partir del análisis de expresión facial. Revista Colombiana de Computación, 19(2), 6-15. https://doi.org/10.29375/25392115.3438

Delgado, D. M., Girón Timaná, D. F., Chanchí, G. E., \& Márceles, K. (2019). Estimación del atributo satisfacción en test de usuarios a partir del análisis de la expresión facial. Revista Ingenierías Universidad de Medellín, 19(36), 13-28. https://doi. org/10.22395/rium.v19n36a1 
Dzulfiqar, M. D., Khairani, D., \& Wardhani, L. K. (2019, March 25). The Development of University Website using User Centered Design Method with ISO 9126 Standard. 2018 6th International Conference on Cyber and IT Service Management, CITSM 2018. https://doi.org/10.1109/ CITSM.2018.8674325

Enriquez, J. G., \& Casas, S. (2013). Usabilidad en aplicaciones móviles. Informe Científico Técnico UNPA, 5(2), 25-47. https://dialnet. unirioja.es/servlet/articulo?codigo $=512352$ $4 \&$ info=resumen\&idioma=SPA

Expósito, C. D., \& Marsollier, R. G. (2020). Virtualidad y educación en tiempos de COVID-19. Un estudio empírico en Argentina. Educación y Humanismo, 22(39), 1-22. https://doi.org/10.17081/ eduhum.22.39.4214

Ferreira, J. M., Acuña, S. T., Dieste, O., Vegas, S., Santos, A., Rodríguez, F., \& Juristo, N. (2020). Impact of usability mechanisms: An experiment on efficiency, effectiveness and user satisfaction. Information and Software Technology, 117, 106195. https://doi. org/10.1016/j.infsof.2019.106195

Finstad, K. (2010). The usability metric for user experience. Interacting with Computers, 22(5), 323-327. https://doi.org/10.1016/j. intcom.2010.04.004

García, F. J., Corell, A., Abella, V., \& Grande, M. (2020). Online assessment in higher education in the time of COVID-19. Education in the Knowledge Society, 21, 1-26. https://doi.org/10.14201/eks.23013

González, J.-L., Montero, F., \& Gutiérrez, F.L. (2012). Evolución del concepto de usabilidad como indicador de la calidad del software. El Profesional de La Información, 21(5), 529-536. https://doi.org/10.3145/ epi.2012.sep.13
Jabbar, A., Gauci, C. G., \& Anstead, C. A. (2021). Parasitology Education Before and After the COVID-19 Pandemic. In Trends in Parasitology (Vol. 37, Issue 1, pp. 3-6). Elsevier Ltd. https://doi.org/10.1016/j. pt.2020.10.009

Jordan, K., David, R., Phillips, T., \& Pellini, A. (2021). Education during the Covid-19 crisis: Opportunities and constraints of using EdTech in low-income countries. Revista Eduación a Distancia, 21(65), $1-15$.

Kem-Mekah, O. (2020). Enseñanza en línea durante la crisis del COVID-19 en la educación universitaria camerunesa: logros y desafíos. Revista Ehquidad, 14, 57-74. https://revistas.proeditio.com/ ehquidad/article/view/3793

Ling, Y., \& Chen, Y. (2011). Enhance the usability of cartographic visualization system by user-centered interface design. Proceedings - 4th International Congress on Image and Signal Processing, CISP 2011, 4, 2061-2065. https://doi. org/10.1109/CISP.2011.6100572

Macaulay, C., Sloan, D., Jiang, X., Forbes, P., Loynton, S., Swedlow, J. R., \& Gregor, P. (2009). Usability and user-centered design in scientific software development. IEEE Software, 26(1), 96-102. https://doi. org/10.1109/MS.2009.27

Martínez, J., \& Garcés, J. (2020). Competencias digitales docentes y el reto de la educación virtual derivado de la COVID-19. Revista Educación y Humanismo, 22(39), 1-16.

Martins, V. F., De Paiva Guimaraes, M., \& Correa, A. G. (2013). Usability test for Augmented Reality applications. Proceedings of the 2013 39th Latin American Computing Conference, CLEI 2013. https://doi. org/10.1109/CLEI.2013.6670668 
Murillo, F. J., \& Duk, C. (2020). El Covid-19 y las Brechas Educativas. Revista Latinoamericana de Educación Inclusiva, 14(1), 11-13. https://doi.org/10.4067/ s0718-73782020000100011

Nielsen, J. (2000). Why You Only Need to Test with 5 Users. Nielsen Norman Group. https://www.nngroup.com/articles/whyyou-only-need-to-test-with-5-users/

Retnani, W. E. Y., Prasetyo, B., Prayogi, Y. P., Nizar, M. A., \& Abdul, R. M. (2018). Usability testing to evaluate the library's academic web site. Proceedings of the 2017 4th International Conference on Computer Applications and Information Processing Technology, CAIPT 2017, 2018-January, 1-4. https://doi.org/10.1109/ CAIPT.2017.8320714

Santos, C., Novais, T., Ferreira, M., Albuquerque, C., De Farias, I. H., \& Furtado, A. P. C. (2016). Metrics focused on usability ISO 9126 based. Iberian Conference on Information Systems and Technologies, CISTI, 2016-July. https://doi.org/10.1109/ CISTI.2016.7521437

UNESCO. (2019). Marco de competencias de los docentes en materia de TIC UNESCO. https://unesdoc.unesco.org/ark:/48223/ pf0000371024

Velásquez, S. M., \& Ruidiaz, K. S. (2020). La educación en tiempo de pandemia COVID 19: ¿realidad o ficción? Revista Cuidarte, 12(1). https://doi.org/10.15649/ cuidarte.1336

Weichbroth, P. (2020). Usability of mobile applications: A systematic literature study. IEEE Access, 8, 55563-55577. https://doi. org/10.1109/ACCESS.2020.2981892
Yaohua, Y., \& Zhengjie, L. (2006). Research on a user-centered design method for interactive online teaching system. International Conference on Communication Technology Proceedings, ICCT. https://doi.org/10.1109/ ICCT.2006.341739

Zeman, J., Tanuska, P., \& Kebisek, M. (2009). The utilization of metrics usability to evaluate the software quality. ICCTD 2009 - 2009 International Conference on Computer Technology and Development, 1, 243-246. https://doi.org/10.1109/ICCTD.2009.58 